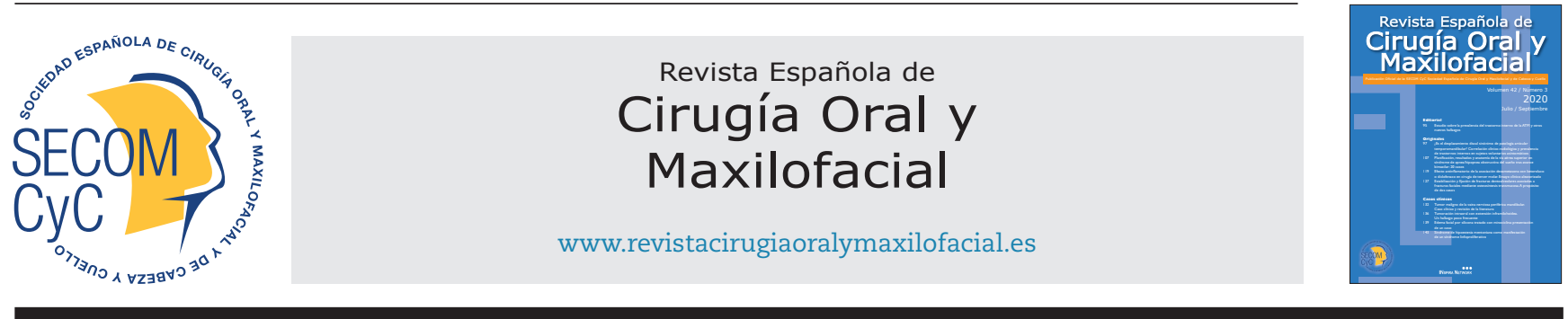

\title{
Original
}

\section{Planificación, resultados y anatomía de la vía aérea superior en síndrome de apnea/hipopnea obstructiva del sueño tras avance bimaxilar: 20 casos}

\author{
Joan Brunsó ${ }^{1}$, Carlos Prol ${ }^{1 *}$, Félix de Carlos², Valentín Cabriada ${ }^{3}$, Josu Mendiola ${ }^{4}$ \\ y Luis Barbier ${ }^{1}$
}

${ }^{1}$ Servicio de Cirugía Maxilofacial, Hospital Universitario Cruces, Barakaldo. Grupo Cirugía Maxilofacial, Instituto de Investigación Sanitaria Biocruces Bizkaia, Barakaldo. Departamento de Estomatología I, Facultad de Medicina y Odontología, Universidad del País Vasco, Leioa, España. ${ }^{2}$ Departamento de Cirugía y Especialidades Médico Quirúrgicas, Universidad de Oviedo, Oviedo, España. ${ }^{3}$ Servicio de Neumología, Hospital Universitario Cruces, Barakaldo, España. ${ }^{4}$ Servicio de Radiodiagnóstico, Hospital Universitario Cruces, Barakaldo, España

INFORMACIÓN DEL ARTÍCULO

\section{Historia del artículo:}

Recibido: 11 de abril de 2020

Aceptado: 15 de junio de 2020

Palabras clave:

Patología del sueño, SAHS, cirugía ortognática, avance maxilomandibular, rotación antihoraria.

\section{R E S U M E N}

Objetivos: El objetivo es presentar el protocolo de trabajo, resultados clínicos y cambios en la vía aérea superior de una cohorte de 20 pacientes con síndrome de apnea/hipopnea obstructiva del sueño, intervenidos de avance maxilomandibular con rotación antihoraria.

Material y métodos: Un comité multidisciplinar determina la indicación quirúrgica, que los pacientes consienten. Se llevan a cabo tomografías computarizadas y polisomnografías antes y después de la cirugía, como protocolo habitual de trabajo. El comité ético de investigación clínica regional aprobó el estudio. Longitudes, volúmenes, área mínima, dimensión anteroposterior, dimensión transversal y otras medidas son determinadas en la vía aérea. El análisis estadístico es descriptivo y comparativo por pares con $\mathrm{p}<0,05$.

Resultados: Los movimientos planificados son 10,40 $\mathrm{mm}$ de avance y 2,11 mm de impactación anterior. El índice de apnea/hipopnea se reduce 30,50 puntos y la saturación de oxígeno capilar periférica mínima aumenta 5,00 puntos. Los resultados clínica y estadísticamente significativos son: $10,98 \mathrm{~mm}$ de acortamiento y $6,26 \mathrm{~mm}^{3}$ de incremento de volumen, especialmente en el compartimento retro-palatal; $91,45 \mathrm{~mm}^{2}, 3,68 \mathrm{~mm}$ y $8,00 \mathrm{~mm}$ de aumento de área, dimensión antero-posterior y dimensión transversal respectivamente; el hioides avanza $1,92 \mathrm{~mm}$.

Conclusiones: El avance maxilomandibular con rotación antihoraria en síndrome de apnea/ hipopnea del sueño moderado-severo logra a corto plazo índice de apnea/hipopnea $<15$ en el $80 \%$ y saturación de oxígeno periférica capilar > 85 en el 75 \% de nuestra serie. Los principales cambios en vía aérea son: acortamiento, incremento de volumen y áreas, forma elíptica y posicionamiento antero-superior del hioides.

\footnotetext{
*Autor para correspondencia:

Correo electrónico: drprolteijeiro@gmail.com (Carlos Prol).
}

DOI: 10.20986/recom.2020.1140/2020

1130-0558/@ 2020 SECOM. Publicado por Inspira Network. Este es un artículo Open Access bajo la licencia CC BY-NC-ND (http:// creativecommons.org/licenses/by-nc-nd/4.0/). 


\section{Planning, results and anatomy of the upper airway in obstructive sleep apnea/hypopnea syndrome after bimaxillary advancement: 20 cases}

\section{A B S T R A C T}

Keywords:

Sleep disolver, OSAHS, orthognatic surgery, maxillo-mandibular advancement, counter clockwise rotation.
Objectives: To present the working protocol, clinical outcomes and upper airway changes of a 20-patient cohort with moderate-severe obstructive sleep apnea/hypopnea syndrome undergoing maxillo-mandibular advancement with counterclockwise rotation.

Material and methods: A multidisciplinary committee determines the surgical indication, which patients consent. Computed tomographies and polysomnographies are performed before and after surgery, as the usual clinical practice protocol. The clinical investigation ethics institutional review board approved the study. Lengths, volumes, minimum area, antero-posterior dimension, transverse dimension and other measurements are determined in the upper airway. Statistical analysis is descriptive and comparative by pairs with $\mathrm{p}<0.05$.

Results: Planned movements are $10.40 \mathrm{~mm}$ of advance and $2.11 \mathrm{~mm}$ of anterior impaction. Apnea/hypopnea index reduces by 30.50 points and minimum peripheral capillary oxygen saturation increases by 5.00 points. Clinically and statistical significant findings are: $10.98 \mathrm{~mm}$ of shortening and $6.26 \mathrm{~mm}^{3}$ of volume enlargement, especially in the retro-palatal compartment; $91.45 \mathrm{~mm}^{2}, 3.68 \mathrm{~mm}$ and $8.00 \mathrm{~mm}$ of area, antero-posterior dimension and transverse dimension widening respectively; hyoid bone advances $1.92 \mathrm{~mm}$.

Conclusions: Maxillo-mandibular advancement with counterclockwise rotation in moderatesevere obstructive sleep apnea/hypopnea syndrome achieves in short-term follow-up apnea/ hypopnea index $<15$ in $80 \%$ and minimum peripheral capillary oxygen saturation $>85$ in $75 \%$ of our series. Main upper airway changes are: shortening, volume and area increase, elliptical shape, and antero-superior hyoid bone movement.

\section{INTRODUCCIÓN}

Los síntomas y consecuencias del síndrome de apnea/ hipopnea obstructiva del sueño (SAHS) son el resultado del colapso repetitivo de la vía aérea superior (VAS). Antes de comenzar cualquier tratamiento conservador o invasivo, el diagnóstico debe ser confirmado y la severidad caracterizada mediante polisomnografía nocturna (PSG) llevada a cabo en un laboratorio especializado. La presión positiva en la vía aérea aplicada de forma continua (CPAP) es el tratamiento de referencia para casos de carácter moderado-severo. En las últimas décadas, los dispositivos de avance mandibular (DAM) también han sido globalmente introducidos. Antes de considerar cualquier procedimiento quirúrgico, es recomendable reevaluar la anatomía, comorbilidades y las preferencias del paciente. Además, la indicación debería ser debatida en un comité multidisciplinar ${ }^{1,2}$. El avance maxilomandibular (AMM), especialmente si se asocia a rotación antihoraria, ensancha la VAS en su conjunto y tensiona los tejidos blandos circundantes. A pesar de ser un tratamiento invasivo, ha sido reportado reducción del índice de apnea/hipopnea (IAH) a valores de la normalidad en el 75-100 \% de casos en el corto a medio plazo de seguimiento ${ }^{1,3-5}$. Se presenta nuestro protocolo de trabajo, la evaluación de resultados clínicos, y un estudio piloto observacional retrospectivo de los cambios morfológicos en la VAS. Se pretende corroborar que nuestros hallazgos, basados en una cohorte de 20 pacientes con SAHS moderado-severo interve- nidos de AMM con rotación antihoraria, son consistentes con la literatura actual.

\section{MATERIAL Y MÉTODOS}

Los criterios de inclusión son: $\geq 18$ años, SAHS moderadosevero en posición no específica o supina, indicación determinada por un comité de patología del sueño (neumología, otorrinolaringología, maxilofacial, odontología, radiodiagnóstico y neurofisiología) y consentimiento informado para la cirugía. Los criterios de exclusión son: mujer embarazada, predominancia de apneas de origen central, deformidades craneofaciales mayores (tales como paladar hendido, maloclusión con asimetría severa, traumatismo o cirugía oncológica de cabeza y cuello) y cirugía distinta al AMM. El protocolo del estudio fue revisado y aprobado por el comité de ética de investigación clínica regional "OSI Ezkerraldea-Enkarterri-Cruces”, código CEIC E19/39, y se adapta a la Declaración de Helsinki. Los pacientes firmaron un consentimiento específico convenientemente informado para el estudio. Se analizan los resultados clínicos y la morfología de VAS, a partir de PSG y tomografía computarizada (TC) como pruebas complementarias de la práctica clínica habitual. Se realizaron un máximo de tres meses antes del AMM y postoperatoriamente entre el tercer mes y el primer año. El diagnóstico, tratamiento y pruebas complementarias tuvieron lugar entre los años 2012 y 2017 en un único centro de tercer nivel. 


\section{Planificación quirúrgica}

Una vez la indicación quirúrgica está establecida, se lleva a cabo un análisis cefalométrico: fotografías faciales e intraorales, telerradiografía lateral de cráneo (TLC), ortopantomografía y modelos de arcadas dentales en yeso ${ }^{6}$. Cuando consideramos al paciente preparado desde un punto de vista de salud bucodental y oclusal, se realiza una TC. A partir de la serie de cortes axiales y modelos dentales actuales, se segmenta una malla dental-craneofacial, que se orienta según el plano natural de la cabeza y datos de la exploración clínica. La osteotomía maxilar tipo Lefort I, la mandibular tipo Obwegeser-Dal Pont y los movimientos pivotados en el incisivo central superior (ICS) son planificados en tres dimensiones (3D); engranando la oclusión más estable, que será la misma que la inicial si no fueron realizados movimientos ortodónticos preoperatorios. Los dispositivos quirúrgicos de reposicionamiento, bien férulas oclusales estándar o guías personalizadas de corte-perforación y placas preformadas, son diseñados y sintetizados mediante tecnología con soporte informático. Otros procedimientos esqueléticos simultáneos también pueden ser trazados y medidos. Las mentoplastias son consideradas para mejorar el perfil facial del paciente y corregir asimetrías frontales, mientras que avances genioglosos se proponen cuando existe un gran colapso retro-glosal (RG) y la anatomía dental-ósea mandibular es favorable ${ }^{7-9}$. El primer autor es el cirujano principal en cuanto a planificación y ejecución de las técnicas quirúrgicas.

\section{Estandarización de pruebas}

Los PSG se llevan a cabo según las recomendaciones de la Sociedad Española de Neumología ${ }^{10}$. Las TC (Philips Brilliance $\mathrm{CT}^{\circledR}, 16$ detectores) son: sin contraste, helicoidales, cortes axiales de $1 \mathrm{~mm}$, con el paciente despierto, en posición supina, quieto, sin tragar, en apnea al final de la espiración, labios relajados, la punta de la lengua en contacto con ICS, la mandíbula en relación céntrica e inclusión completa del hioides y el cráneo.

\section{Datos generales y resultados clínicos}

Se reportan datos epidemiológicos (sexo, etnia y edad en el momento del AMM), datos exploratorios (índice de masa corporal [IMC], escala de Mallampati y clase de Angle), cirugías y ortodoncia previas, procedimientos simultáneos al AMM relacionados con la VAS, y sistema de reposicionamiento de los segmentos maxilares. El avance es determinado en el ICS y en el Pogonion (Pog), el movimiento vertical anterior (MVA) en el ICS, y el movimiento vertical posterior en los primeros molares superiores derecho e izquierdo. Se registra a partir de las PSG el AHI en posición no específica, supino y no supino; y los parámetros basal y mínimo de la saturación periférica de oxígeno capilar (SPO2). SAHS leve se considera con IAH 5-15 y síntomas de somnolencia diurna, moderado con $16-30$, y severo $>30^{1,2}$.

\section{Variables de VAS: (NemoTec FAB ${ }^{\circledR}$ software, versión 2.7.0)}

La malla craneofacial de cada paciente se orienta en el plano axial alineando el conducto naso-palatino con la apófisis odontoides, que es alineada al mismo tiempo con la base de la crista galli en el plano coronal. En el medio-sagital, la referencia principal horizontal es el plano del paladar duro (PD), mientras que la vertical es el plano perpendicular que cruza por el punto más antero-inferior de la segunda vértebra cervical (AIC2) (Figura 1). Planos paralelos al del PD que pasan por la punta de la úvula (PU), punta de epiglotis (PE) y el punto antero-inferior del hioides (AIH) dividen la VAS en tres compartimentos: retropalatal (RP), RG e hipofaringe (HPh). Paralelas a la referencia vertical, longitudes (L) son medidas por compartimentos, el sumatorio y el total de forma independiente. Se segmenta una malla de aire cuyos límites son: superior el plano PD, inferior el plano AIH, anterior tejidos blandos excluyendo el aire de la cavidad oral, posterior la musculatura prevertebral, y lateral la musculatura parafaríngea. Volúmenes (V) se calculan a partir de un punto densidad aire de aproximadamente -1000 HU y un rango de $\pm 500 \mathrm{HU}$, también por compartimentos, el sumatorio y el total independientemente (Figura 2). En cortes axiales paralelos al PD, se determinan el área mínima (AR), su localización y dimensiones (D), entendidas como la distancia recta antero-posterior (AP) y transversal (TV) máxima de densidad aire (Figura 3). Además, distancias horizontales paralelas al plano PD desde la referencia vertical son cuantificadas: la espina nasal posterior (ENP), apófisis geni (AG), AIH y tejidos blandos pre-vertebrales (TBP) a la altura de AIC2 (Figura 4). Todas las medidas son llevadas a cabo por el segundo autor.

\section{Análisis estadístico: (SPSS ${ }^{\oplus}$ software, versión 23.0.0)}

Se calculan estadísticos descriptivos de frecuencias. También se realiza un análisis comparativo por pares de las variables continuas dependientes (pre y postoperatorio). La

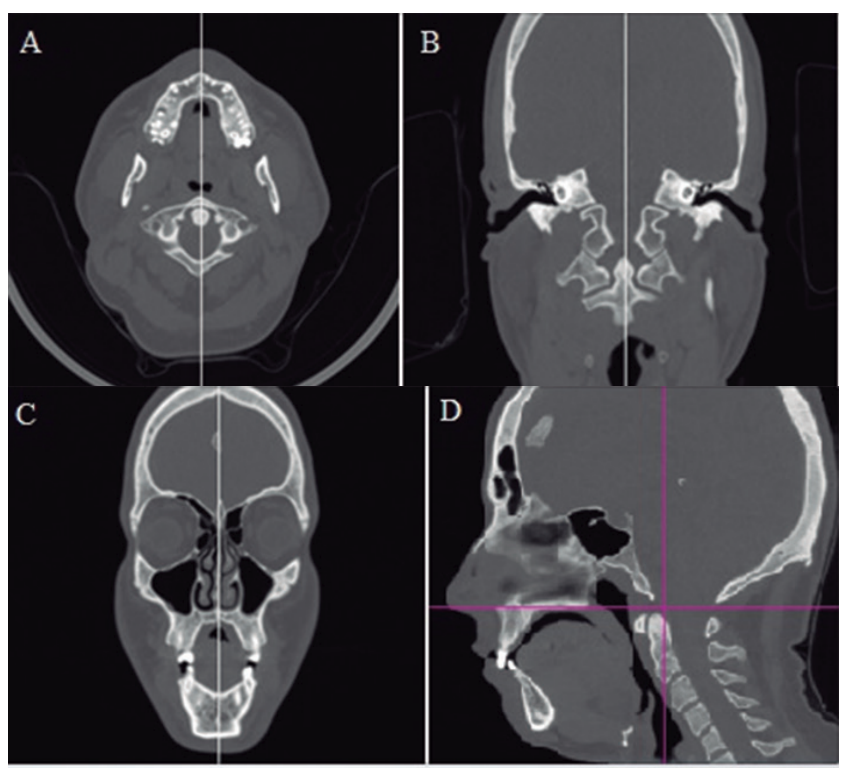

Figura 1. Caso 11 preoperatorio. Método de orientación secuencial en el plano axial (A), coronal (B, C) y mediosagital (D). Plano medio-sagital en blanco y planos de referencia en magenta. 


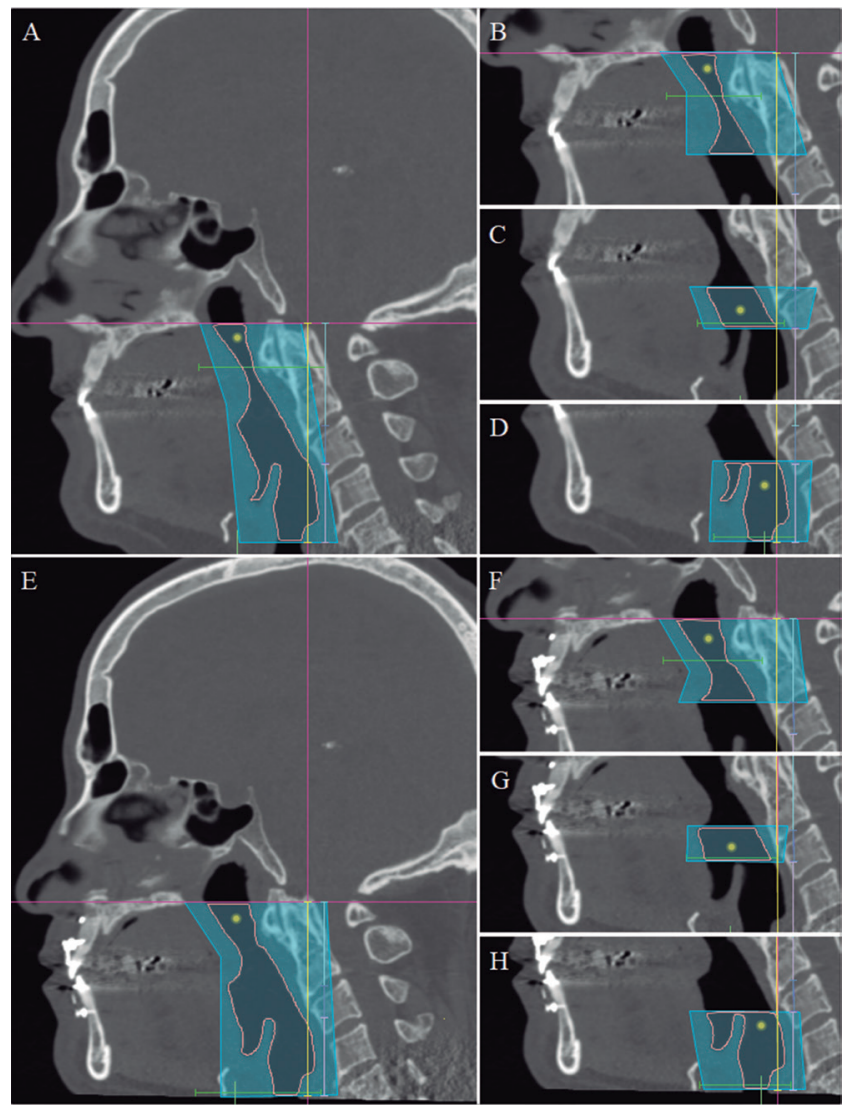

Figura 2. Caso 15 preoperatorio (A-D) y postoperatorio (E-H). Cortes medio-sagitales. Determinación de longitudes verticales y volúmenes. Punto de referencia y longitud total en amarillo. Longitud retro-palatal en turquesa claro, longitud retro-glosal en azul agrisado e hipofaringe en lavanda. Límites del trazado para la estimación de volumen en azul (total y por compartimentos), delimitación de la malla de vía aérea en rosa claro. Localización del área mínima en verde.
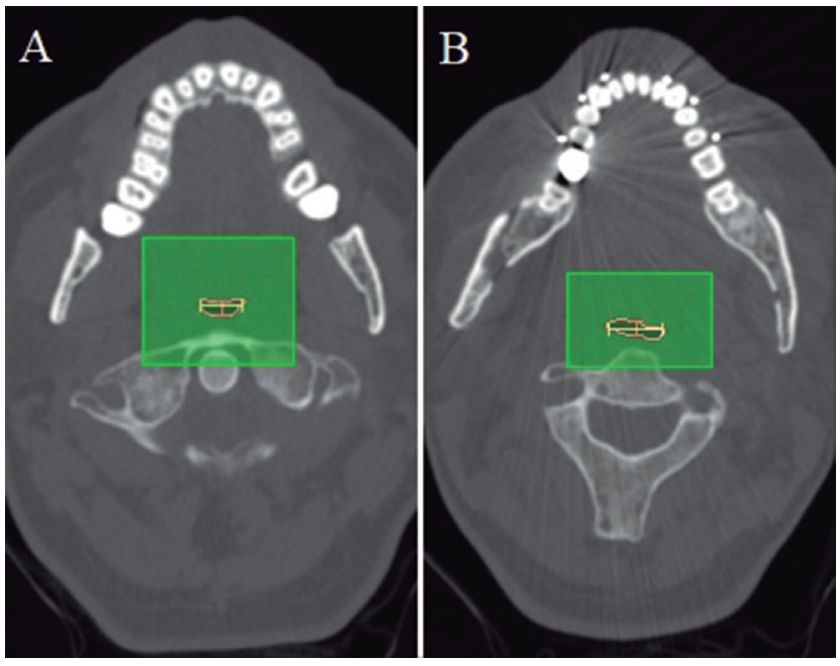

Figura 3. Caso 16 preoperatorio (A) y postoperatorio (B). Corte axial a nivel del área mínima, trazada en rosa claro. Dimensión antero-posterior en rosa oscuro y dimensión transversa en amarillo.

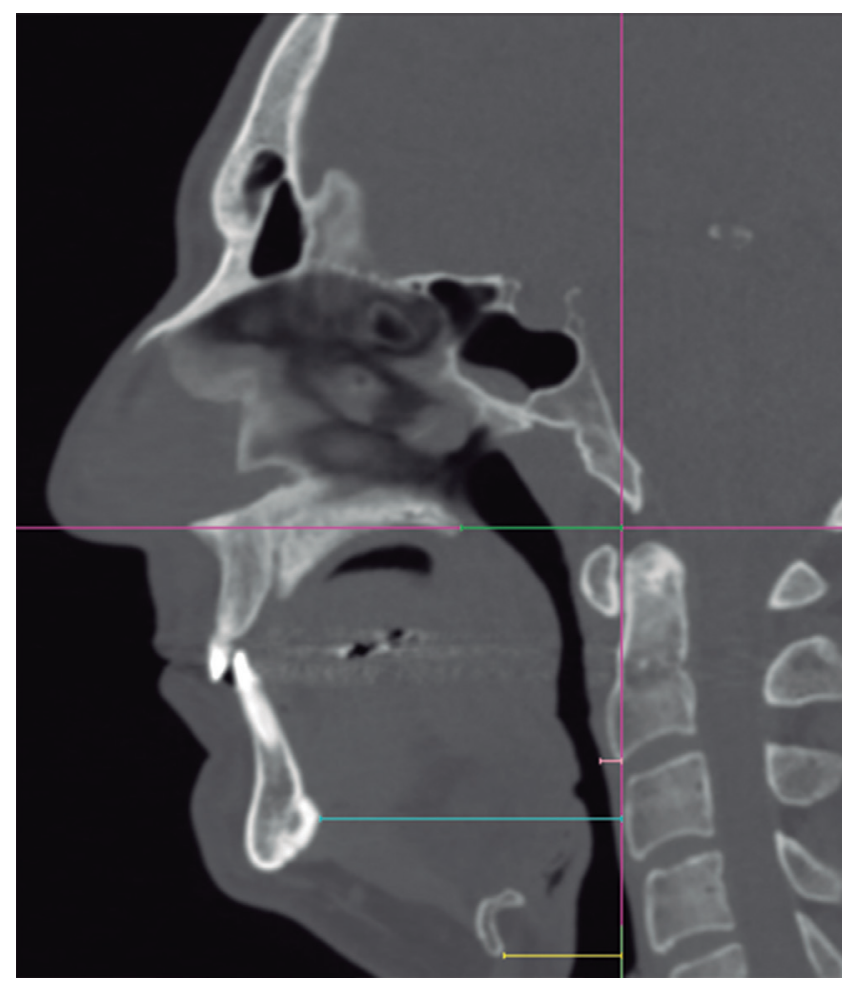

Figura 4. Caso 16 preoperatorio. Corte medio-sagital.

Determinación de longitudes horizontales. Planos de referencia en magenta. Longitud a espina nasal posterior en verde, de tejidos blandos prevertebrales en rosa, a apófisis geni en turquesa y a punto antero-inferior de hioides en amarillo.

normalidad de las variables se verifica con el test de Kolmogorov-Smirnov, por tanto el test paramétrico ( $t$-Student para muestras dependientes) o no paramétrico (rangos de Wilcoxon para muestras dependientes) correspondiente es aplicado, con $\mathrm{p}<0,05$.

\section{RESULTADOS}

El ratio masculino/femenino es 19/1. El $100 \%$ son blanco caucásicos, con edad media de 41 años (32-52). El $45 \%$ tiene IMC > 25, el $85 \%$ Mallampati III-IV y el $45 \%$ sin maloclusión alguna. El $60 \%$ no había sido sometido a cirugías relacionadas con la VAS previo al AMM y el 85 \% tampoco realizó ortodoncia preoperatoria. En cinco pacientes se llevó a cabo mentoplastia y uno avance geniogloso simultáneos al AMM. Todas las cirugías cursaron sin incidentes intraoperatorios. El reposicionamiento de los maxilares es con férulas oclusales en el $20 \%$ de los casos, mientras que en el resto se realiza con sistemas personalizados de guías y placas. El avance planificado medio en ICS es 10,40 mm (9,70-11,00), con 2,11 mm (7,00-1,00) de MVA y nivelación posterior, así se lleva cabo la rotación antihoraria de forma que el Pog es proyectado anteriormente 14,60 mm $(11,30-22,30)$ (Tabla I).

El IAH global se reduce significativamente 30,50 puntos de media después de la cirugía, mientras que en posición supina 
51,00 puntos. Esto supone IAH $<15$ en el $80 \%$ y < 5 en el $50 \%$ postoperatoriamente. Hay algunos datos ausentes en posición supina y no supina si el paciente mantuvo la misma posición durante la PSG. Aunque la SPO2 basal no cambia, la mínima mejora 5,00 puntos de media, es decir, que es $>85 \%$ en el $75 \%$ de casos (Tabla II).

Los cambios en VAS clínica y estadísticamente significativos incluyen la reducción de L total 10,98 mm de media, especialmente en la región $\mathrm{RP}$, aunque disminuye en todos los compartimentos. El V total aumenta $6,26 \mathrm{~mm}^{3}$ de media, doblándose en la región RP y sin significación estadística en $\mathrm{HPh}$. El rango de error entre la diferencia del total y sumatorio es $<1 \mathrm{~mm}$ en cuanto a $\mathrm{L} \mathrm{y}<1 \mathrm{~mm}^{3}$ respecto al V. El AR mínima, localizada en RP en el $85 \%$ de TC preoperatorios y en el $55 \%$ de los post, se incrementa $49,72 \mathrm{~mm}^{2}$ de media, prácticamente triplicándose. La D-TV se ensancha más que la D-AP, sugiriendo una expansión en forma de elipse, con diferencias de 8,67 mm y 3,62 mm de media, respectivamente. Hay un incremento significativo de las L horizontales, a destacar la L-AIH, que implica una posición más anterior del hioides. También asciende, debido al acortamiento vertical en conjunto de la VAS. A pesar de que la reducción de L-TBP es estadísticamente significativa, no lo consideramos clínicamente porque la diferencia es $<1$ $\mathrm{mm}$ de media y mediana (Tabla III).

\section{DISCUSIÓN}

Según el protocolo quirúrgico por fases de Standford, el AMM formaba inicialmente parte de la fase II de manejo ${ }^{11,12}$. Se consideraba cuando otros procedimientos quirúrgicos habían fallado, principalmente enfocados a localizaciones específicas de la VAS. Actualmente es considerado como tratamiento primario, como un abordaje de cirugía única con impacto global en VAS, en pacientes con deformidades dentofaciales predisponentes al colapso tal y como retrognatia, hipoplasia maxilar o resalte incisal aumentado. Será un tratamiento secundario en caso de intolerancia a CPAP o DAM ${ }^{4,5}$. El AMM pretende ensanchar las regiones RP y RG mediante tracción antero-superior de la ENP y AG, inserciones de la musculatura velofaríngea y suprahioidea. Además, también tensiona la musculatura parafaríngea ${ }^{13}$. En casos refractarios, existe ya una fase III de Standford que incluye la neuroestimulación del hipogloso ${ }^{14}$. A pesar de que ha sido demostrado que el IAH mejora claramente en SAHS tras el AMM, la mayoría de estudios publicados tiene una presentación muy heterogénea de planificaciones, detalles de la técnica, resultados clínicos y evaluación morfológica de VAS ${ }^{15}$.

En cuanto a planificación, igual que en otros casos quirúrgicos de deformidades dentofaciales sin SAHS, se deben considerar tres pilares básicos: oclusión, estética y VAS. La práctica clínica actual y habitual es trabajar con radiología 3D digitalizada, la cual proporciona más información y permite un mayor control y precisión que el 2D, especialmente en los planos frontales ${ }^{6,9}$. La tomografía por excelencia para evaluar los huesos craneofaciales es la de haz de cono (CBCT). Sin embargo, pensamos que es más fidedigno un TC médico helicoidal en pacientes SAHS, ganando más resolución de tejidos blandos y posiblemente mayor precisión en la segmentación de VAS también, a costa de más exposición a radiación. Estas cirugías tienen un gran impacto en la salud y aspectos psicosociales por la cosmética implícita, por lo que CBCT/TC se consideran no solo para planificar, sino también para el análisis cefalométrico inicial y el control postoperatorio, logrando una evaluación integral 3D ${ }^{16}$. Nosotros realizamos el primer control con TC en pacientes SAHS, pero no el estudio inicial o controles sucesivos.

Debemos tener en cuenta diferencias en SAHS comparando con población general que se somete a cirugía ortognática. Los pacientes SAHS son habitualmente adultos de edad media en la cuarta-quinta década, con compensaciones posicionales corporales para facilitar el paso de aire como hiperextensión y traslación cervical anterior, más riesgo anestésico, más factores de riesgo cardiovascular y más comorbilidades cardiacas, respiratorias y neurológicas ${ }^{2}$. Existe una tendencia a la birretrusión maxilar y atrofia ósea con la edad debido a la pérdida de la capa ósea esponjosa, haciendo las osteotomías más complejas. Los perfiles faciales tienden a ser convexos con mayor laxitud de tejido blando, tolerando la tracción relativamente bien, pero menos predecible en cuanto a cambios cosméticos con las estimaciones cefalométricas estándar y de comportamiento de tejidos blandos faciales, que están basadas en población de adultos jóvenes ${ }^{17}$. Con respecto a la salud bucodental, muchos pacientes SAHS son respiradores orales y existe un mayor desgaste dental, más reconstrucciones coronales, más implantes dentales, más sectores edéntulos, mayores compensaciones, peor estado periodontal con mayor riesgo de retracciones gingivales y pérdidas de vitalidad pulpar. En cuanto a dispositivos ortodónticos, pacientes SAHS pueden a veces no precisar o no desear llevar a cabo movimientos dentales, de forma que no ocurrirían cambios en la oclusión pre y postoperatoria. Sin embargo, habrá situaciones donde para garantizar una oclusión estable será imprescindible una mínima descompensación, coordinación de arcadas y tallados. En todos los casos se colocarán unos arcos rígidos con anclajes fuertes antes del procedimiento quirúrgico, de cara a mantener las fijaciones intermaxilares durante el posicionamiento de los fragmentos osteotomizados con sistemas convencionales de férulas y para realizar ajustes ortodónticos postoperatorios, que es necesario prácticamente siempre ${ }^{18}$.

El movimiento de AMM debería ser el máximo que permita estabilidad oclusal, suficiente contacto óseo entre los segmentos osteotomizados para lograr consolidación, vascularización garantizada de tejidos blandos después de su despegamiento y tracción, y parámetros cefalométricos razonables. Una complicación habitualmente referida son los perfiles protrusivos, aunque suelen ser favorablemente aceptados debido a su connotación rejuvenecedora ${ }^{17}$.

En cuanto a particularidades de la técnica, a veces el maxilar superior necesita ser segmentado en varios fragmentos en caso de hipoplasias trasversas. Nosotros realizamos una segmentación en forma de H. Si la discrepancia es mayor de 5-8 mm, preferiríamos la técnica de expansión rápida palatina asistida quirúrgicamente, posponiendo el AMM a un segundo tiempo (caso 5). En presencia de narices pequeñas preoperatorias, consideramos osteotomías de Lefort I sub-espinales, intentando preservar la musculatura perinasal y modificar menos la base y punta nasales. Las osteotomías mandibulares a nivel de la cortical bucal no deberían ser muy posteriores, al menos entre el segundo y primer molar, para asegurar suficiente contacto 


\section{Tabla I. Datos generales y movimientos planificados}

\begin{tabular}{|c|c|c|c|c|c|c|c|c|c|c|c|c|c|c|c|}
\hline Caso & $\begin{array}{l}\text { Edad } \\
\text { (años) }\end{array}$ & Etnia & Sexo & IMC & Mallampati & $\begin{array}{l}\text { Clase } \\
\text { Angle }\end{array}$ & $\begin{array}{c}\mathrm{Sx} \\
\text { (previa) }\end{array}$ & $\begin{array}{l}\text { Ortho } \\
\text { (previa) }\end{array}$ & $\begin{array}{c}\text { Sx } \\
\text { (actual) }\end{array}$ & $\begin{array}{l}\text { Osteotomía/ } \\
\text {-síntesis }\end{array}$ & $\begin{array}{c}\mathrm{Av}_{-} \\
\mathrm{ICS}(\mathrm{mm})\end{array}$ & $\begin{array}{c}\text { MVA_ } \\
\text { ICS (mm) }\end{array}$ & $\begin{array}{c}\text { MVP }_{-} \\
1.6(\mathrm{~mm})\end{array}$ & $\begin{array}{c}\mathrm{MVP}_{-} \\
2.6(\mathrm{~mm})\end{array}$ & $\begin{array}{c}\mathrm{Av}_{-} \\
\text {Pog }(\mathrm{mm})\end{array}$ \\
\hline 01 & 50 & Blanco-Cau & M & 33,00 & III & I & No & No & $\mathrm{AMM}+$ rot anti & Estándar & 11,0 & Estándar & 11,0 & 1,2 & 12,0 \\
\hline 02 & 39 & Blanco-Cau & M & 21,28 & II & II & $\begin{array}{l}\text { Septoplastia, } \\
\text { adenoidectomia }\end{array}$ & No & $\mathrm{AMM}+$ rot anti & & & $-1,0$ & 4,1 & 3,9 & 16,1 \\
\hline 03 & 34 & Blanco-Cau & M & 28,76 & III & II & No & Sí & $\mathrm{AMM}+$ rot anti & Personalizado & 10,0 & $-2,0$ & 1,0 & 1,0 & 14,0 \\
\hline 04 & 48 & Blanco-Cau & M & 23,78 & IV & I & No & No & $\mathrm{AMM}+$ rot anti & Personalizado & 10,0 & $-1,0$ & 0,0 & 0,0 & 12,6 \\
\hline 05 & 45 & Blanco-Cau & M & 19,87 & III & II & SARPE & Sí & $\mathrm{AMM}+$ rot anti & Personalizado & 10,0 & $-2,0$ & 3,0 & 3,0 & 16,8 \\
\hline 06 & 42 & Blanco-Cau & F & 32,52 & III & I & No & No & $\mathrm{AMM}+$ rot anti & Personalizado & 10,5 & $-1,0$ & 0,0 & 0,1 & 13,1 \\
\hline 07 & 37 & Blanco-Cau & M & 27,06 & IV & I & No & No & $\mathrm{AMM}+$ rot anti & Personalizado & 10,5 & $-1,0$ & 1,0 & 1,0 & 13,3 \\
\hline 08 & 37 & Blanco-Cau & M & 22,76 & IV & II & Uvulopalatofaringoplastia & No & $\mathrm{AMM}+$ rot anti & Personalizado & 10,0 & $-2,0$ & 2,2 & 2,0 & 12,7 \\
\hline 09 & 36 & Blanco-Cau & M & 21,88 & I & III & No & No & $\begin{array}{l}\text { AMM + rot anti, } \\
\text { mentoplastia }\end{array}$ & Personalizado & 11,0 & $-1,0$ & 3,0 & 3,3 & 16,8 \\
\hline 10 & 32 & Blanco-Cau & M & 28,37 & IV & II & No & No & $\begin{array}{l}\text { AMM + rot anti, } \\
\text { mentoplastia }\end{array}$ & Personalizado & 10,0 & $-2,0$ & 3,0 & 3,0 & 17,0 \\
\hline 11 & 45 & Blanco-Cau & M & 23,99 & IV & II & No & No & $\mathrm{AMM}+$ rot anti & Personalizado & 11,0 & $-1,0$ & 1,0 & 1,2 & 12,9 \\
\hline 12 & 45 & Blanco-Cau & M & 25,06 & IV & II & Amigdalectomía & No & $\mathrm{AMM}+$ rot anti & Estándar & 11,0 & $-2,0$ & 2,1 & 2,0 & 14,0 \\
\hline 13 & 41 & Blanco-Cau & M & 25,35 & II & II & Adenoidectomía & No & $\mathrm{AMM}+$ rot anti & Personalizado & 9,7 & $-4,2$ & 2,9 & 2,7 & 11,3 \\
\hline 14 & 38 & Blanco-Cau & M & 27,00 & III & I & No & No & $\begin{array}{l}\text { AMM + rot anti } \\
\text { mentoplastia }\end{array}$ & Estándar & 10,0 & $-7,0$ & 2,0 & 2,0 & 13,7 \\
\hline 15 & 41 & Blanco-Cau & M & 36,11 & IV & I & Adenoidectomía & No & $\mathrm{AMM}+$ rot anti & Personalizado & 10,3 & $-1,9$ & 0,9 & 1,0 & 12,5 \\
\hline 16 & 43 & Blanco-Cau & M & 29,48 & III & I & No & No & $\mathrm{AMM}+$ rot anti & Personalizado & 11,0 & $-3,0$ & 1,0 & 1,0 & 12,7 \\
\hline 17 & 52 & Blanco-Cau & M & 26,83 & IV & I & No & No & $\begin{array}{l}\text { AMM + rot anti, } \\
\text { mentoplastia }\end{array}$ & Personalizado & 11,0 & $-2,5$ & 2,0 & 2,0 & 18,6 \\
\hline 18 & 32 & Blanco-Cau & M & 23,88 & IV & II & Septoplastia & Sí & $\begin{array}{l}\text { AMM + rot anti } \\
\text { mentoplastia }\end{array}$ & Personalizado & 10,0 & $-2,5$ & 3,0 & 3,0 & 22,3 \\
\hline 19 & 48 & Blanco-Cau & M & 24,16 & IV & II & No & No & $\mathrm{AMM}+$ rot anti & Personalizado & 10,0 & $-2,0$ & 0,0 & 0,7 & 12,4 \\
\hline 20 & 44 & Blanco-Cau & M & 23,77 & IV & I & CENS & No & $\begin{array}{l}\text { AMM + rot anti, } \\
\text { Av geniogloso }\end{array}$ & Personalizado & 10,0 & $-2,0$ & $-1,0$ & 0,0 & 14,6 \\
\hline
\end{tabular}

1.6: primer molar superior derecho. 2.6: primer molar superior izquierdo. AMM: avance maxilomandibular. Av: avance. Cau: caucásico. CENS: cirugía endoscópica nasosinusal. F: femenino. ICS: incisivo central superior. IMC: índice de masa corporal. M: masculino. mm: milímetros. MVA: movimiento vertical anterior. MVP: movimiento vertical posterior. Ortho: ortodoncia. Pog: Pogonion. rot anti: rotación antihoraria. SARPE: expansión rápida palatina asistida quirúrgicamente. Sx: cirugía. 
Tabla II. Polisomnografías

\begin{tabular}{|c|c|c|c|c|c|c|c|c|c|c|}
\hline Caso & $\begin{array}{l}\text { IAH } \\
\text { _PRE }\end{array}$ & $\begin{array}{l}\text { IAH } \\
\text { _POST }\end{array}$ & $\begin{array}{c}\text { IAH_SUP } \\
\text { _PRE }\end{array}$ & $\begin{array}{c}\text { IAH_SUP } \\
\text { _POST }\end{array}$ & $\begin{array}{c}\mathrm{IAH} \_\mathrm{NSUP} \\
\text { _PRE }\end{array}$ & $\begin{array}{c}\text { IAH_NSUP } \\
\text { _POST }\end{array}$ & $\begin{array}{c}\text { SPO2_BAS } \\
\text { _PRE }\end{array}$ & $\begin{array}{c}\text { SPO2_BAS } \\
\text { _POST }\end{array}$ & $\begin{array}{l}\text { SPO2_MIN } \\
\text { _PRE }\end{array}$ & $\begin{array}{c}\text { SPO2_MIN } \\
\text { _POST }\end{array}$ \\
\hline 01 & 37 & 23 & 37 & 41 & 37 & 5 & 95 & 95 & 89 & 87 \\
\hline 02 & 68 & 5 & 67 & 7 & 73 & 0 & 95 & 95 & 82 & 83 \\
\hline 03 & 31 & 7 & 59 & 12 & 11 & 1 & 98 & 97 & 93 & 90 \\
\hline 04 & 33 & 0 & 48 & 0 & 31 & 0 & 92 & 94 & 92 & 86 \\
\hline 05 & 28 & 9 & 57 & 10 & 11 & 4 & 96 & 97 & 85 & 92 \\
\hline 06 & 14 & 3 & 21 & 4 & 12 & 2 & 96 & 93 & 88 & 85 \\
\hline 07 & 29 & 2 & 50 & 2 & 9 & 0 & 94 & 95 & 81 & 85 \\
\hline 08 & 35 & 9 & 35 & 14 & - & 6 & 96 & 97 & 85 & 92 \\
\hline 09 & 61 & 16 & 61 & 16 & - & - & 96 & 96 & 77 & 91 \\
\hline 10 & 85 & 8 & 88 & 8 & 69 & - & 94 & 95 & 63 & 82 \\
\hline 11 & 76 & 0 & 87 & 0 & 36 & 0 & 93 & 95 & 79 & 88 \\
\hline 12 & 51 & 1 & 51 & 1 & - & 0 & 97 & 98 & 85 & 90 \\
\hline 13 & 15 & 0 & 28 & 0 & 1 & 0 & 97 & 97 & 80 & 96 \\
\hline 14 & 22 & 0 & - & 0 & 22 & 0 & 96 & 96 & 89 & 92 \\
\hline 15 & 72 & 35 & 89 & 35 & 39 & - & 94 & 97 & 86 & 77 \\
\hline 16 & 34 & 1 & 35 & 2 & 33 & 0 & 94 & 95 & 86 & 91 \\
\hline 17 & 33 & 25 & 73 & 30 & 9 & 12 & 93 & 93 & 85 & 86 \\
\hline 18 & 40 & 3 & 50 & 4 & 35 & 0 & 94 & 94 & 88 & 91 \\
\hline 19 & 67 & 5 & 67 & 6 & - & 5 & 96 & 96 & 87 & 91 \\
\hline 20 & 33 & 2 & 56 & 2 & 8 & - & 96 & 96 & 82 & 92 \\
\hline Media & 43,20 & 7,70 & 55,74 & 9,70 & 27,25 & 2,19 & 95.10 & 95.55 & 84.10 & 88.35 \\
\hline Mediana & 34,50 & 4,00 & 56,00 & 5,00 & 26,50 & 0,00 & 95.50 & 95.50 & 85.00 & 90.00 \\
\hline sd & 21,03 & 9,74 & 19,79 & 12,17 & 21,24 & 3,41 & 1.55 & 1.39 & 6.47 & 4.46 \\
\hline $\mathrm{K}-\mathrm{S} \mathrm{p}$ & 0,015 & 0,002 & 0,879 & 0,018 & 0,083 & 0,000 & 0.013 & 0.250 & 0.027 & 0.047 \\
\hline tow p & \multicolumn{2}{|c|}{0,000} & \multicolumn{2}{|c|}{0,000} & \multicolumn{2}{|c|}{0,003} & \multicolumn{2}{|c|}{0,099} & \multicolumn{2}{|c|}{0,019} \\
\hline
\end{tabular}

BAS: basal. IAH: índice de apnea/hipopnea. K-S: test Kolmogorov-Smirnov. MIN: mínimo. NSUP: posición no supina. p: significación. POST: postoperatorio. PRE: preoperatorio. sd: desviación estándar. SPO2: saturación

de oxígeno capilar. SUP: posición supina. t: test t-Student para muestras dependientes. W: test rangos de Wilcoxon para muestras dependientes. 


\section{Tabla III. Longitudes verticales (en $\mathrm{mm}$ ), volúmenes (en $\mathrm{mm}^{3}$ ), áreas (en $\left.\mathrm{mm}^{2}\right)$, dimensiones (en $\mathrm{mm}$ ) y longitudes horizontales (en $\mathrm{mm}$ )}

\begin{tabular}{|c|c|c|c|c|c|c|c|c|c|c|c|c|c|c|c|c|}
\hline Caso & $\begin{array}{l}\text { L_RP } \\
\text { _PRE }\end{array}$ & $\begin{array}{c}\text { L_RP } \\
\text { _POST }\end{array}$ & $\begin{array}{l}\text { L_RG } \\
\text { _PRE }\end{array}$ & $\begin{array}{l}\text { L_RG } \\
\text { _POST }\end{array}$ & $\begin{array}{c}\text { L_HPh } \\
\text { _PRE }\end{array}$ & $\begin{array}{l}\text { L_HPh } \\
\text { _POST }\end{array}$ & $\begin{array}{c}\text { L_TOT } \\
\text { _PRE }\end{array}$ & $\begin{array}{l}\text { L_TOT } \\
\text { _POST }\end{array}$ & $\begin{array}{l}\text { V_RP } \\
\text { _PRE }\end{array}$ & $\begin{array}{l}\text { V_RP } \\
\text { _POST }\end{array}$ & $\begin{array}{l}\text { V_RG } \\
\text { _PRE }\end{array}$ & $\begin{array}{l}\text { V_RG } \\
\text { _POST }\end{array}$ & $\begin{array}{c}\text { V_HPh } \\
\text { _PRE }\end{array}$ & $\begin{array}{l}\text { V_HPh } \\
\text { _POST }\end{array}$ & $\begin{array}{c}\text { V_TOT } \\
\text { _PRE }\end{array}$ & $\begin{array}{l}\text { V_TOT } \\
\text { _POST }\end{array}$ \\
\hline 01 & 31,87 & 34,89 & 17,83 & 18,09 & 31,08 & 31,02 & 80,48 & 83,99 & 5.10 & 10,36 & 5,45 & 7,10 & 5,63 & 8,40 & 16,29 & 25,68 \\
\hline 02 & 31,28 & 27,53 & 30,37 & 20,65 & 22,96 & 25,56 & 84,86 & 73,74 & 1,77 & 6,19 & 0,59 & 3,55 & 3,20 & 2,79 & 5,94 & 12,68 \\
\hline 03 & 33,09 & 29,93 & 19,72 & 14,64 & 42,25 & 32,20 & 95,76 & 76,77 & 1,40 & 2,26 & 3,02 & 4,42 & 6,43 & 4,71 & 10,81 & 12,19 \\
\hline 04 & 39,14 & 27,78 & 10,67 & 20,25 & 32,02 & 26,62 & 81,84 & 74,65 & 3,82 & 5,19 & 2,88 & 6,63 & 8,41 & 6,01 & 15,31 & 18,23 \\
\hline 05 & 32,30 & 26,45 & 21,20 & 15,58 & 28,26 & 29,66 & 81,76 & 71,69 & 0,60 & 7,39 & 3,03 & 7,27 & 9,91 & 9,87 & 13,13 & 26,13 \\
\hline 06 & 39,95 & 36,84 & 7,61 & 9,32 & 26,64 & 20,42 & 73,71 & 66,57 & 3,72 & 7,75 & 1,59 & 2,79 & 7,77 & 4,45 & 13,10 & 15,31 \\
\hline 07 & 31,25 & 31,20 & 25,26 & 18,72 & 23,93 & 26,38 & 80,44 & 76,66 & 4,59 & 8,41 & 2,59 & 4,03 & 4,57 & 7,25 & 11,89 & 19,27 \\
\hline 08 & 22,01 & 22,15 & 39,23 & 24,71 & 28,71 & 26,41 & 89,95 & 74,13 & 1,71 & 4,11 & 6,05 & 7,57 & 4,73 & 3,93 & 12,50 & 15,71 \\
\hline 09 & 31,60 & 30,38 & 16,68 & 8,68 & 30,72 & 23,87 & 78,13 & 62,93 & 1,70 & 6,47 & 2,13 & 2,66 & 4,91 & 10,02 & 8,51 & 19,40 \\
\hline 10 & 35,75 & 16,63 & 13,01 & 14,67 & 31,57 & 31,78 & 79,77 & 63,06 & 2,24 & 0,86 & 1,81 & 4,05 & 8,23 & 11,81 & 12,24 & 18,84 \\
\hline 11 & 41,71 & 30,00 & 20,85 & 11,41 & 29,01 & 29,58 & 91,58 & 70,56 & 4,82 & 10,31 & 2,96 & 6,14 & 6,98 & 17,01 & 15,03 & 34,02 \\
\hline 12 & 31,99 & 27,21 & 20,43 & 13,61 & 26,21 & 21,77 & 79,07 & 62,59 & 3,03 & 8,56 & 2,38 & 3,20 & 6,58 & 5,79 & 11,86 & 17,33 \\
\hline 13 & 31,77 & 26,35 & 15,11 & 10,98 & 24,73 & 25,04 & 71,45 & 62,37 & 3,73 & 6,88 & 1,98 & 3,14 & 5,92 & 8,27 & 11,73 & 18,52 \\
\hline 14 & 31,97 & 23,82 & 20,04 & 20,84 & 27,60 & 26,80 & 79,72 & 71,46 & 4,23 & 7,38 & 2,84 & 7,07 & 7,36 & 8,92 & 14,37 & 24,40 \\
\hline 15 & 42,25 & 36,52 & 15,45 & 13,46 & 32,25 & 34,11 & 90,40 & 84,25 & 5,56 & 12,67 & 5,02 & 7,70 & 7,27 & 10,50 & 18,02 & 31,36 \\
\hline 16 & 38,71 & 38,09 & 17,35 & 17,87 & 33,38 & 28,69 & 89,44 & 84,65 & 3,02 & 4,09 & 1,21 & 2,06 & 5,40 & 6,50 & 9,69 & 12,33 \\
\hline 17 & 34,56 & 27,28 & 15,26 & 11,26 & 33,22 & 27,28 & 83,04 & 65,83 & 8,95 & 10,63 & 7,19 & 5,64 & 16,55 & 9,94 & 33,04 & 26,55 \\
\hline 18 & 36,33 & 24,48 & 35,02 & 34,91 & 15,76 & 14,97 & 87,11 & 74,35 & 5,68 & 4,06 & 2,56 & 6,44 & 4,13 & 2,73 & 12,33 & 13,55 \\
\hline 19 & 33,48 & 27,49 & 14,03 & 11,16 & 35,29 & 33,48 & 82,79 & 71,96 & 7,62 & 11,98 & 2,70 & 4,61 & 11,42 & 16,46 & 22,11 & 33,67 \\
\hline 20 & 31,41 & 27,73 & 29,82 & 24,44 & 36,74 & 35,25 & 97,45 & 86,95 & 8,89 & 8,68 & 3,73 & 13,91 & 9,36 & 15,83 & 22,13 & 38,74 \\
\hline Media & 34,12 & 28,64 & 20,25 & 16,76 & 29,62 & 27,54 & 83,94 & 72,96 & 4,11 & 7,21 & 3,09 & 5,5 & 7,24 & 8,56 & 14,50 & 21,70 \\
\hline Mediana & 32,70 & 27,63 & 18,78 & 15,13 & 29,87 & 27,04 & 82,32 & 72,85 & 3,78 & 7,39 & 2,77 & 5,13 & 6,78 & 8,34 & 12,80 & 19,06 \\
\hline sd & 4,66 & 5,21 & 8,09 & 6,40 & 5,66 & 4,97 & 6,84 & 7,74 & 2,38 & 3,16 & 1,66 & 2,70 & 3,01 & 4,28 & 5,90 & 7,97 \\
\hline $\mathrm{K}-\mathrm{S} p$ & 0,013 & 0,155 & 0,030 & 0,200 & 0,200 & 0,200 & 0,200 & 0,200 & 0,200 & 0,200 & 0,001 & 0,200 & 0,200 & 0,200 & 0,044 & 0,018 \\
\hline tow $p$ & \multicolumn{2}{|c|}{0,000} & \multicolumn{2}{|c|}{0,012} & \multicolumn{2}{|c|}{0,017} & \multicolumn{2}{|c|}{0,000} & \multicolumn{2}{|c|}{0,000} & \multicolumn{2}{|c|}{0,000} & \multicolumn{2}{|c|}{0,136} & \multicolumn{2}{|c|}{0,000} \\
\hline
\end{tabular}


Tabla III (Cont.). Longitudes verticales (en $\mathbf{m m}$ ), volúmenes (en $\mathbf{~ m m}^{3}$ ), áreas (en $\mathbf{~ m m}^{2}$ ), dimensiones (en $\mathbf{m m}$ ) y longitudes horizontales (en $\mathbf{m m}$ )

\begin{tabular}{|c|c|c|c|c|c|c|c|c|c|c|c|c|c|c|}
\hline Caso & $\begin{array}{l}\text { AR_MIN } \\
\text { _PRE }\end{array}$ & $\begin{array}{c}\text { AR_MIN } \\
\text { _POST }\end{array}$ & $\begin{array}{c}\text { D-AP_MIN } \\
\text { _PRE }\end{array}$ & $\begin{array}{c}\text { D-AP_MIN } \\
\text { _POST }\end{array}$ & $\begin{array}{c}\text { D-TV_MIN } \\
\text { _PRE }\end{array}$ & $\begin{array}{c}\text { D-TV_MIN } \\
\text { _POST }\end{array}$ & $\begin{array}{l}\text { L_ENP } \\
\text { _PRE }\end{array}$ & $\begin{array}{l}\text { L_ENP } \\
\text { _POST }\end{array}$ & $\begin{array}{l}\text { L-TBP } \\
\text { _PRE }\end{array}$ & $\begin{array}{l}\text { L_TBP } \\
\text { _POST }\end{array}$ & $\begin{array}{l}\text { L_AG } \\
\text { _PRE }\end{array}$ & $\begin{array}{l}\text { L_AG } \\
\text { _POST }\end{array}$ & $\begin{array}{l}\text { L_AIH } \\
\text { _PRE }\end{array}$ & $\begin{array}{l}\text { L_AIH } \\
\text { _POST }\end{array}$ \\
\hline 01 & 71,91 & 142,09 & 5,04 & 8,23 & 16,50 & 22,78 & 50,75 & 63,73 & 6,86 & 7,75 & 69,04 & 81,40 & 41,61 & 45,87 \\
\hline 02 & 0,00 & 50,29 & 0,00 & 4,47 & 0,00 & 18,33 & 37,79 & 44,89 & 5,32 & 5,86 & 54,27 & 58,55 & 31,92 & 34,41 \\
\hline 03 & 0,00 & 0,00 & 0,00 & 0,00 & 0,00 & 0,00 & 40,37 & 39,94 & 6,34 & 5,20 & 60,56 & 73,84 & 29,57 & 32,85 \\
\hline 04 & 36,29 & 111,54 & 4,69 & 7,94 & 10,32 & 18,04 & 43,32 & 43,85 & 7,12 & 5,79 & 64,04 & 67,70 & 28,46 & 27,78 \\
\hline 05 & 0,00 & 229,19 & 0,00 & 8,22 & 0,00 & 26,91 & 38,34 & 41,49 & 5,07 & 4,96 & 52,58 & 64,70 & 24,32 & 23,71 \\
\hline 06 & 42,80 & 119,60 & 6,11 & 9,98 & 9,40 & 14,67 & 49,55 & 47,42 & 6,17 & 4,88 & 73,61 & 72,79 & 25,70 & 28,85 \\
\hline 07 & 53,19 & 168,07 & 5,91 & 10,91 & 8,45 & 18,63 & 41,20 & 47,33 & 5,32 & 4,42 & 75,79 & 83,79 & 35,23 & 34,06 \\
\hline 08 & 26,77 & 58,41 & 2,65 & 5,52 & 11,50 & 13,17 & 42,82 & 51,24 & 6,70 & 7,67 & 67,94 & 78,39 & 42,10 & 44,14 \\
\hline 09 & 25,99 & 121,75 & 2,80 & 8,25 & 10,32 & 18,05 & 29,79 & 50,88 & 5,27 & 6,08 & 54,42 & 77,69 & 33,36 & 38,63 \\
\hline 10 & 19,25 & 1,21 & 3,83 & 1,00 & 6,70 & 1,10 & 43,83 & 56,64 & 6,40 & 5,87 & 63,56 & 78,70 & 23,89 & 32,14 \\
\hline 11 & 32,35 & 261,75 & 4,12 & 7,64 & 6,87 & 29,12 & 36,33 & 49,88 & 4,99 & 5,07 & 62,11 & 77,32 & 30,83 & 33,80 \\
\hline 12 & 27,20 & 120,75 & 4,16 & 6,54 & 10,16 & 18,60 & 44,16 & 45,76 & 6,22 & 5,44 & 61,30 & 69,84 & 23,54 & 28,12 \\
\hline 13 & 52,51 & 210,06 & 4,92 & 12,30 & 13,41 & 21,24 & 34,53 & 45,43 & 6,41 & 6,15 & 64,12 & 69,84 & 27,94 & 28,55 \\
\hline 14 & 73,78 & 233,50 & 6,87 & 11,56 & 13,75 & 27,15 & 47,04 & 48,74 & 5,54 & 4,68 & 59,21 & 66,78 & 25,11 & 30,20 \\
\hline 15 & 46,25 & 140,25 & 4,64 & 10,86 & 12,06 & 14,81 & 44,20 & 53,36 & 5,91 & 5,29 & 76,32 & 86,01 & 34,98 & 37,96 \\
\hline 16 & 48,66 & 61,94 & 4,11 & 4,25 & 12,34 & 17,02 & 32,91 & 36,57 & 5,34 & 5,17 & 62,74 & 68,19 & 28,48 & 30,57 \\
\hline 17 & 159,50 & 313,25 & 10,57 & 13,79 & 20,66 & 37,14 & 54,20 & 53,87 & 6,28 & 4,76 & 79,00 & 76,66 & 27,38 & 29,88 \\
\hline 18 & 33,50 & 100,50 & 3,86 & 5,12 & 10,61 & 14,85 & 33,69 & 36,81 & 5,25 & 4,99 & 49,46 & 49,42 & 13,57 & 11,33 \\
\hline 19 & 99,18 & 253,33 & 5,74 & 15,10 & 21,19 & 19,76 & 40,94 & 52,57 & 5,43 & 4,40 & 67,87 & 75,56 & 29,41 & 29,70 \\
\hline 20 & 61,80 & 146,64 & 5,09 & 5,88 & 15,48 & 31,78 & 32,37 & 41,02 & 5,77 & 5,36 & 55,19 & 76,26 & 20,17 & 27,71 \\
\hline Media & 45,55 & 142,21 & 4,26 & 7,88 & 10,49 & 19,16 & 40,91 & 47,57 & 5,89 & 5,49 & 63,66 & 72,67 & 28,88 & 31,51 \\
\hline Mediana & 39,55 & 131,00 & 4,40 & 8,08 & 10,47 & 18,47 & 41,07 & 47,38 & 5,84 & 5,25 & 63,15 & 74,70 & 28,47 & 30,39 \\
\hline$s d$ & 37,09 & 86,81 & 2,48 & 3,95 & 5,93 & 8,94 & 6,55 & 6,79 & 0,64 & 0,91 & 8,31 & 8,68 & 6,73 & 7,30 \\
\hline$K-S p$ & 0,139 & 0,200 & 0,082 & 0,200 & 0,200 & 0,200 & 0,787 & 0,990 & 0,183 & 0,127 & 0,532 & 0,500 & 0,712 & 0,033 \\
\hline tow $p$ & \multicolumn{2}{|c|}{0,000} & \multicolumn{2}{|c|}{0,000} & \multicolumn{2}{|c|}{0,000} & \multicolumn{2}{|c|}{0,000} & \multicolumn{2}{|c|}{0,030} & \multicolumn{2}{|c|}{0,000} & \multicolumn{2}{|c|}{0,002} \\
\hline
\end{tabular}

AG: apófisis geni. AIH: anteroinferior hioides. AR: área. D-AP: dimensión anteroposterior. D-TV: dimensión transuersal. ENP: espina nasal posterior. HPh: hipofaringe. K-S: test Kolmogorou-Smirnou test. L: longitud. p: significación. MIN: mínimo. POST: postoperatorio. PRE: preoperatorio. RG: retro-glosal. RP: retro-palatal. sd: desviación estándar. t: test t-Student para muestras dependientes. TBP: tejidos blandos pre-vertebrales. TOT: total. V: volumen. W: test rangos de Wilcoxon para muestras dependientes. 
óseo durante la osteosíntesis. Cuando los fragmentos osteotomizados son traccionados anteriormente, las interferencias óseas y adhesiones de tejidos blandos deben ser eliminadas, con cuidado de no comprometer la vascularización. Resaltamos la liberación completa, pero no ligadura, de los pedículos palatinos mayores en el maxilar superior, mientras que en la mandíbula destaca el despegamiento del periostio en la cortical basilar y de la cincha pterigo-maseterina preservando únicamente su inserción posterior.

La rotación antihoraria potencia el AMM, ensanchando la región RP todavía más y también la RG por el reposicionamiento mandibular más antero-superior con el máximo impacto óseo facial en el Pog, compensando situaciones en la cuales no alcanzamos los 10-12 $\mathrm{mm}$ recomendados de avance en el ICS $^{19}$. Se debe tener precaución con los gaps posteriores en el maxilar superior, requiriendo en ocasiones injertos óseos $u$ osteosíntesis adicional en la arbotante maxilomalar. Defectos palpables pueden ocurrir en el borde basilar mandibular. Existe también riesgo, especialmente si no se realizó ortodoncia preoperatoria, de exposición incisal disminuida y sonrisa gingival posterior ${ }^{20}$.

El método tradicional de reposicionamiento de los fragmentos maxilares osteotomizados es por medio de férulas oclusales. Han sido desarrollados sistemas de navegación y de guías y placas personalizadas en la última década. $\mathrm{Su}$ uso puede reducir tiempos quirúrgicos y proporcionar mejor control de la dimensión vertical y posición condilar. Se requiere certeza absoluta de que los registros preoperatorios de relación céntrica mandibular son todos correctos para el diseño y síntesis de estos dispositivos personalizados. Una desventaja es el mayor gasto económico que conllevan. Podría estar justificado en pacientes SAHS, debido a los grandes movimientos requeridos, que implican una estabilización de fragmentos más compleja que otros casos de cirugía ortognática convencional. Nosotros llevamos a cabo las osteotomías maxilares primero con estos sistemas, mientras que preferimos empezar los procedimientos por la mandíbula con férulas oclusales, de modo que la férula intermedia no sea muy voluminosa por la mordida abierta generada con la rotación antihoraria ${ }^{7,8}$.

Otros procedimientos complementarios con implicaciones en la VAS pueden ser realizados utilizando el mismo abordaje intraoral del AMM. Septoplastias y turbinectomías tratan de mejorar el flujo aéreo a través de la cavidad nasal (CN). Remodelado óseo y ensanchamiento de las aperturas piriformes se puede llevar a cabo antes de completar la osteosíntesis maxilar, con precaución de no comprometer la arbotante maxilonasal y la premaxilar, donde las placas y tornillos serán situados. Los avances genioglosos y mentoplastias de deslizamiento altas (Figura 5), además de mejorar la estética facial, proyectan todavía más la AG traccionando anterosuperiormente los músculos geniogloso y genihioideo. Estas dos técnicas deberían siempre realizarse después de haber completado el AMM. Las uvulopalatofaringoplastias asociadas a AMM tienen riesgo de incompetencia velofaríngea ${ }^{13}$. Por lo tanto, las escisiones deberían ser muy conservadoras, solo en pacientes con claro exceso de tejido blando, siempre antes del AMM, y avance limitado por perfiles convexos protrusivos que son más frecuentes en etnias asiáticas o negroides que en blanco-caucásicos ${ }^{20}$.
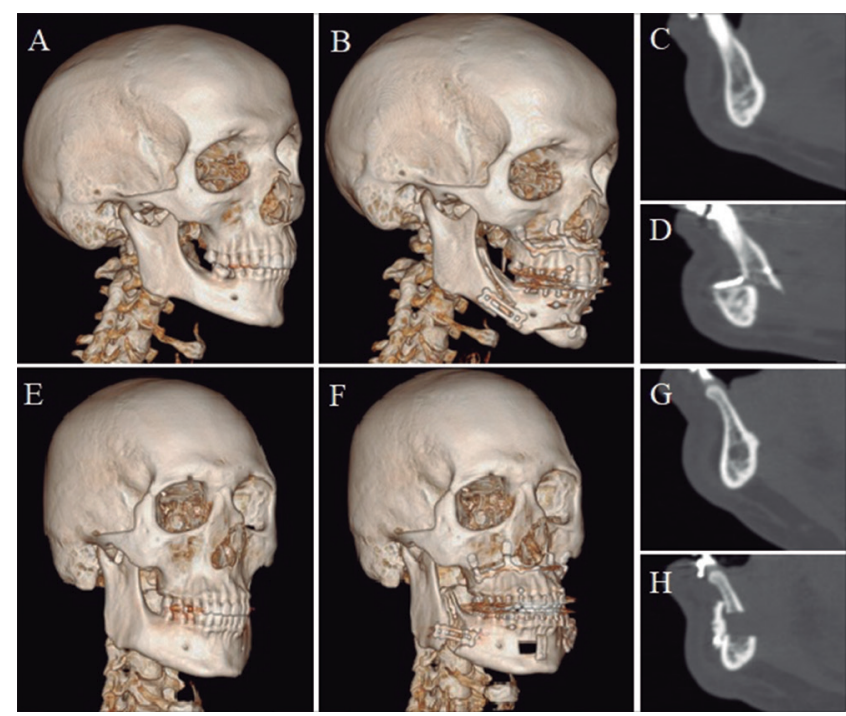

Figura 5. Casos 17 (A-D) y 20 (E-H). Mallas craneofaciales y detalles en corte medio-sagital. Preoperatorio y postoperatorio, avance maxilomandibular con rotación antihoraria asociado a mentoplastia y avance geniogloso.

Con respecto a la morfología de VAS tras el AMM, varios estudios han reportado cuantitativamente cambios en $\mathrm{CN}$, nasofaringe (NPh), RP, RG e HPh. Los hallazgos más destacables se localizan en RP y RG y en relación con la posición del hioides ${ }^{19,21}$. CN, cavidad oral y NPh son excluidos en nuestro análisis debido a la tremenda variabilidad anatómica y compleja delimitación de márgenes en esas localizaciones. Las principales limitaciones radican en la estandarización de pruebas (TLC, CBCT, TC o resonancia magnética [RM]), el método de segmentación 3D y la adquisición de medidas, con frecuencia pobremente especificada y difícilmente reproducible $^{22}$. En general, se toman las mismas referencias para el diagnóstico cefalométrico, la planificación de movimientos y las medidas de VAS: plano de Frankfurt, posición natural de la cabeza y vertical gravitacional verdadera ${ }^{23-25}$. Sin embargo, los tejidos blandos posteriores contienen la VAS, que puede modificar su morfología por los siguientes movimientos de la columna cervical: flexo-extensión, traslación, rotación e inclinaciones transversales. Es una limitación crítica, ya que aunque las pruebas radiológicas estuvieran perfectamente estandarizadas, el posicionamiento cervical no está asegurado. Una máscara craneofacial cervical es una posible solución, pero no hemos encontrado ningún estudio que trabaje con ello. Otra opción es utilizar una metodología de puntos clave de referencia 3D (tanto de estructuras óseas como de tejidos blandos) que proporcione una segmentación fiable y medidas precisas volumétricas, de áreas de sección transversal y distancias lineales. Reportes previos como el presente estudio utilizan este tipo de metodología, pero hay que destacar que el nuestro es con TC médicos en posición supina mientras los demás son con CBCT y pacientes habitualmente sentados ${ }^{22-25}$.

Destacan los siguientes estudios comparativos prepostoperatorio en pacientes SAHS, con método similar al nuestro y también consistentes con nuestros resultados. Faria y cols. ${ }^{23}$ reportan mediante RM un incremento de $\mathrm{V}$ del $26,72 \%$ en RP y 
del 27,20 \% en Rg. El mismo grupo a partir de TLC concluye que cada $\mathrm{mm}$ de avance implica una ganancia RP de 0,76 mm y RG de 1,20 mm; y también comunica una posición más superior de hioides. Zinser y cols. ${ }^{24}$, utilizando TC, presentan resultados significativos de: acortamiento de VAS, aumento de V, forma más elíptica y una posición más anterosuperior del hioides. El mayor impacto es en la región RP, RG y en la localización del AR mínima. Schendel y cols. ${ }^{25}$ reportan datos significativos de incremento de $\mathrm{V}$ de x 2,5 globalmente, x 3,5 en RP y x 1,5 en RG; y una arquitectura más elíptica. El acortamiento de VAS y la elevación del hioides no son significativos.

Algunos autores hacen un intento de correlacionar y cuantificar la cantidad de avance planificado en relación directa con los cambios de L, V, AR y D $\mathrm{D}^{19,21,23}$. A pesar del gran interés de estas estimaciones, no fueron considerados en nuestra serie. Están involucradas muchas particularidades anatómicas individuales y la planificación de cada paciente es única. Es más, los movimientos óseos reales muy rara vez son exactos a los planificados, aun con sistemas personalizados y especialmente en la mandíbula. Creemos que un mayor número de pacientes debería ser reclutado para realizar esta clase de análisis y precaución extra debería ser tomada en cuenta en su interpretación, en tanto que las herramientas informáticas de estimación de los cambios en tejidos blandos de la cara y en VAS con la reposición dental-ósea no están claramente establecidas ni validadas hoy día ${ }^{7,8}$.

Otras estructuras distintas a la VAS en pacientes SAHS pueden ser segmentadas 3D, estudiadas y comparadas, tal y como el maxilar superior, mandíbula, incluso tejidos blandos (lengua, paladar blando o TBP) sabiendo que sus mallas son menos precisas que las de aire u óseas. Técnicas dinámicas de imagen, diferenciando la posición supina de no supina e incluso vigilia frente a estado inducido de sueño, podrían proporcionar más información relevante en cuanto a la colapsabilidad. Por la gran cantidad de CBCT llevados a cabo en la actualidad de cara a la evaluación clínica dental y maxilofacial, un paquete específico de medidas radiológicas 3D junto con datos de la exploración física (IMC especialmente) podría sugerir perfiles de alto riesgo de SAHS antes de que los síntomas aparecieran, como una herramienta complementaria a los cuestionarios de somnolencia diurna ${ }^{17,19,22}$.

Aunque los IAH no están en rango de la normalidad, todos los pacientes de nuestra serie describen en la entrevista clínica menor ronquido, mejoría de la somnolencia diurna y buena funcionalidad laboral. Nuestro estudio compara PSG y TC en el corto plazo postoperatorio como limitación. Sin embargo, el SAHS es una enfermedad crónica y requiere manejo personalizado y multidisciplinar con seguimiento a largo plazo. El AMM proporciona calidad de vida con mejoría de aspectos clínicos y reducción de morbilidad, pero no cura el síndrome. Tras el AMM, parece haber estabilidad esquelética con el tiempo, pero no está bien establecida en términos de tejidos blandos y sintomatología, que tiende a recurrir inherente al envejecimiento ${ }^{1,2,19}$.

\section{CONCLUSIONES}

La técnica de AMM con rotación antihoraria para el tratamiento de pacientes SAHS moderado-severo, debido al repo- sicionamiento anterior del maxilar y la proyección anterosuperior de la mandíbula, tiene un alto porcentaje de éxito en el corto plazo de seguimiento. Logra IAH global < 15 en el $80 \%$ y SPO2 mínima > 85 en el $75 \%$ de nuestra serie. El mayor avance y rotación posibles son planificados, teniendo en cuenta limitaciones oclusales, óseas, de tejido blando y cosméticas. Cambios anatómicos en VAS tras el AMM estadísticamente significativos y clínicamente relevantes son acortamiento e incremento de $\mathrm{V}$ total, principalmente a costa del compartimento RP, que es la localización más colapsable. El AR mínima se triplica, la arquitectura de VAS es más elíptica y el hioides se mueve a una posición más anterosuperior. La estabilidad de estos cambios morfológicos y clínicos a largo plazo está todavía por determinar. Más pacientes se seguirán reclutando para el presente estudio.

\section{CONFLICTOS DE INTERÉS}

Los autores no declaran conflictos de interés.

\section{FINANCIACIÓN}

El presente estudio fue financiado, el autor para correspondencia (y segundo) Carlos Prol recibió la beca "BBK-BIOCRUCES BIZKAIA Post-MIR Curso 2019-2020".

\section{B I B L I O G R A F Í A}

1. Epstein LJ, Kristo D, Strollo PJ, Friedman N, Malhotra A, Patil SP, et al. Clinical guideline for the evaluation, management and long-term care of obstructive sleep apnea in adults. J Clin Sleep Med. 2009;5(3):263-76.

2. Lloberes P, Durán-Cantolla J, Martínez-García MÁ, Marín JM, Ferrer A, Corral J, et al. Diagnosis and treatment of sleep apnea-hypopnea syndrome. Archivos de Bronconeumología (English Edition). 2011;47(3):143-56. DOI: 10.1016/S15792129(11)70034-9.

3. Ronchi P, Novelli G, Colombo L, Valsecchi S, Oldani A, Zucconi $\mathrm{M}$, et al. Effectiveness of maxillo-mandibular advancement in obstructive sleep apnea patients with and without skeletal anomalies. Int J Oral Maxillofac Surg. 2010;39(6):541-7. DOI: 10.1016/j.ijom.2010.03.006.

4. Zaghi S, Holty JE, Certal V, Abdullatif J, Guilleminault C, Powell NB, et al. Maxillomandibular Advancement for Treatment of Obstructive Sleep Apnea: A Meta-analysis. JAMA Otolaryngol Head Neck Surg. 2016;142(1):58-66. DOI: 10.1001/jamaoto.2015.2678.

5. Holty JE, Guilleminault C. Maxillomandibular advancement for the treatment of obstructive sleep apnea: a systematic review and meta-analysis. Sleep Med Rev. 2010;14(5):287-97. DOI: 10.1016/j.smrv.2009.11.003.

6. Birbe J. Planificación clásica en cirugía ortognática. Rev Esp Cir Oral Maxilofac. 2014;36:99-107. DOI: 10.1016/j.maxilo.2012.04.007.

7. Brunso J, Franco M, Constantinescu T, Barbier L, Santamaría JA, Alvarez J. Custom-Machined Miniplates and Bone-Supported Guides for Orthognathic Surgery: A New Surgical Procedure. J Oral Maxillofac Surg. 2016;74(5):1061.e1-.e12. DOI: 10.1016/j. joms.2016.01.016.

8. Brunso J, Prol C, Franco M, Carlos Fd, Martin JC, Santamaria JA. Guías y miniplacas personalizadas: un protocolo guiado para 
cirugía ortognática. Rev Esp Cir Oral Maxilofac. 2017;39(1):7-14. DOI: 10.1016/j.maxilo.2016.05.001.

9. Aboul-Hosn Centenero S. Planificación tridimensional y utilización de férulas Computer Aided Design/Computed Aided Manufacturing en cirugía ortognática. Rev Esp Cir Oral Maxilofac. 2014;36(3):108-12. 10.1016/j.maxilo.2013.02.003

10. Martín Viñé L, Manchado García N, Pamiés Solé M, Bello, Sebastián J, Ordax Carvajo E. Polisomnografía: procedimientos. In: Morante Vélez F, Ordax Carbajo E, editors. Manual Separ de Procedimientos 20 Manual de Procedimientos en trastornos respiratorios del sueño: Novartis Farmacéutica SA; 2010. p. 21-32.

11. Riley RW, Powell NB, Guilleminault C. Obstructive sleep apnea syndrome: a review of 306 consecutively treated surgical patients. Otolaryngol Head Neck Surg. 1993;108(2):117-25. DOI: 1 $0.1177 / 019459989310800203$

12. Riley RW, Powell NB, Guilleminault C. Obstructive sleep apnea syndrome: a surgical protocol for dynamic upper airway reconstruction. J Oral Maxillofac Surg. 1993;51(7):742-7. DOI: 10.1016/s0278-2391(10)80412-4.

13. Aurora RN, Casey KR, Kristo D, Auerbach S, Bista SR, Chowdhuri S, et al. Practice parameters for the surgical modifications of the upper airway for obstructive sleep apnea in adults. Sleep. 2010;33(10):1408-13. DOI: 10.1093/sleep/33.10.1408.

14. Liu SY, Riley RW. Continuing the Original Stanford Sleep Surgery Protocol From Upper Airway Reconstruction to Upper Airway Stimulation: Our First Successful Case. J Oral Maxillofac Surg. 2017;75(7):1514-8. DOI: 10.1016/j.joms.2017.02.008.

15. Caples SM, Rowley JA, Prinsell JR, Pallanch JF, Elamin MB, Katz SG, et al. Surgical modifications of the upper airway for obstructive sleep apnea in adults: a systematic review and metaanalysis. Sleep. 2010;33(10):1396-407. DOI: 10.1093/ sleep/33.10.1396.

16. Shaheen E, Shujaat S, Saeed T, Jacobs R, Politis C. Three-dimensional planning accuracy and follow-up protocol in orthognathic surgery: a validation study. Int J Oral Maxillofac Surg. 2019;48(1):71-6. DOI: 10.1016/j.ijom.2018.07.011.

17. Neelapu BC, Kharbanda OP, Sardana HK, Balachandran R, Sardana V, Kapoor P, et al. Craniofacial and upper airway morphology in adult obstructive sleep apnea patients: A system- atic review and meta-analysis of cephalometric studies. Sleep Med Rev. 2017;31:79-90. DOI: 10.1016/j.smrv.2016.01.007.

18. Larson BE. Orthodontic preparation for orthognathic surgery. Oral Maxillofac Surg Clin North Am. 2014;26(4):441-58. DOI: 10.1016/j.coms.2014.08.002.

19. Louro RS, Calasans-Maia JA, Mattos CT, Masterson D, CalasansMaia MD, Maia LC. Three-dimensional changes to the upper airway after maxillomandibular advancement with counterclockwise rotation: a systematic review and meta-analysis. Int J Oral Maxillofac Surg. 2018;47(5):622-9. DOI: 10.1016/j. ijom.2017.11.003.

20. Wei S, Zhang Y, Guo X, Yu W, Wang M, Yao K, et al. Counterclockwise maxillomandibular advancement: a choice for Chinese patients with severe obstructive sleep apnea. Sleep Breath. 2017;21(4):853-60. DOI: 10.1007/s11325-017-1484-7.

21. Tan SK, Leung WK, Tang ATH, Zwahlen RA. How does mandibular advancement with or without maxillary procedures affect pharyngeal airways? An overview of systematic reviews. PLoS One. 2017;12(7):e0181146. DOI: 10.1371/journal. pone.0181146.

22. Neelapu BC, Kharbanda OP, Sardana HK, Gupta A, Vasamsetti $\mathrm{S}$, Balachandran $\mathrm{R}$, et al. The reliability of different methods of manual volumetric segmentation of pharyngeal and sinonasal subregions. Oral Surg Oral Med Oral Pathol Oral Radiol. 2017;124(6):577-87. DOI: 10.1016/j.00oo.2017.08.020.

23. Faria AC, da Silva-Junior SN, Garcia LV, dos Santos AC, Fernandes MR, de Mello-Filho FV. Volumetric analysis of the pharynx in patients with obstructive sleep apnea (OSA) treated with maxillomandibular advancement (MMA). Sleep Breath. 2013;17(1):395-401. DOI: 10.1007/s11325-012-0707-1.

24. Zinser MJ, Zachow S, Sailer HF. Bimaxillary 'rotation advancement' procedures in patients with obstructive sleep apnea: a 3-dimensional airway analysis of morphological changes. Int J Oral Maxillofac Surg. 2013;42(5):569-78. DOI: 10.1016/j. ijom.2012.08.002.

25. Schendel SA, Broujerdi JA, Jacobson RL. Three-dimensional upper-airway changes with maxillomandibular advancement for obstructive sleep apnea treatment. Am J Orthod Dentofacial Orthop. 2014;146(3):385-93. DOI: 10.1016/j.ajodo.2014.01.026. 